\title{
Entity of Stage 4 CKD over 75 Years Old is Different from that of Less than 75 Years of Age: Characteristics of Elderly Patients with Stage 4 CKD Suzuki $\mathrm{H}^{1 *}$, Inoue $\mathrm{T}^{1}$, Kikuta $\mathrm{T}^{1}$, Hamada $\mathrm{Y}^{\mathbf{2}}$ and Okada $\mathrm{H}^{1}$
}

${ }^{1}$ Department of Nephrology, Saitama Medical University, Japan

${ }^{2}$ Community Health Science Center, Japan

\begin{abstract}
Background: Although age had a strong effect on the risk of development to end-stage renal disease (ESRD), it is reported that stage 4 CKD patients aged $>75$ years were less likely to subsequently commence renal replacement therapy (RRT) than those aged $<75$ years. In the present study, the longitudinal follow-up of elderly patients with stage 4 CKD was carried out in a single center in order to evaluate the prognosis of the patients $>75$ years in comparison with
\end{abstract} those between 74 and 65 years and to characterize patients with stage 4 CKD older than 75 years.

Methods: This was a prospective, observational, single-center cohort study. Patients were recruited from specialist renal clinics from January 2004 to December 2005. All participating patients were followed for 5 years or until death, commencement of dialysis therapy, receiving renal transplantation, finding of neoplasm, or occurrence of a cardiovascular event. We utilized electronic databases to determine the incidence of RRT and morbidity.

Results: The underlying renal disease of CKD included diabetic nephropathy (DM) (91 patients, $51 \%$ ) chronic glomerulonephritis (GN) (41 patients, 23\%), hypertensive nephrosclerosis (HT) (44 patients, $25 \%$ ), and other diseases (3 patients, 1\%) in the younger group (74 and 65 years)and DM (24 patients, $23 \%)$, GN (24 patients, 23\%), HT(54 patients, $51 \%$ ), and other diseases (4 patients, $4 \%$ ) in the elderly population (older than 75 years). A proportion of the underlying renal diseases were significantly differences between the two groups. In the elderly group, the prevalence of diabetic nephropathy was significantly lower $(P<0.01)$ and that of HT was significantly higher $(P<0.01)$. The incidencefree rates at 1 year and 3 years were $90 \%$ and $81 \%$ in the elderly group and those were $90 \%$ and $57 \%$ in the younger group, respectively. In the patients without diabetic nephropathy, introduction to RRT was significantly lower compared with those with diabetic nephropathy in the younger group $(P<0.05)$. The incidence-free rates at 1 year and 3 years were $88 \%$ and $80 \%$ in the patients without diabetic nephropathy and those were $87 \%$ and $38 \%$ in the patients with diabetic nephropathy, respectively. In the late referral patients, the prevalence of diabetic nephropathy were significantly higher and that of $\mathrm{GN}$ and $\mathrm{HT}$ were significantly lower compared with those of the non-late referral patients $(\mathrm{P}<0.01)$.

Conclusions: From these findings, it is concluded that in the patients $>75$ years, the values of eGFR may be overestimated and a prognosis of diabetic nephropathy was poor. Besides, the early nephrology referral is encouraged.

Keywords: eGFR; Late nephrology referral; Diabetic nephropathy; Renal replacement therapy

\section{Introduction}

The proportion of older people in the general population is steadily increasing and the fastest growing segment of that population during the past decade is older than 75 years. With this increases in prevalence of elderly people, proportion of prevalent chronic kidney disease (CKD) increased in parallel $[1,2]$. In spite of these situations, the appropriate treatment of older patients with CKD remains unclear. Moreover, there are a lot of unresolved problems in real world [3]. For example, are there any differences in underlying renal diseases in CKD patients $<75$ and $>75$ years?; what are the age-related risk factors for progression to end-stage renal disease (ESRD) and incidence of cardiovascular disease (CVD) [4,5]; is a chance of more cohesive multidisciplinary team approach to $\mathrm{CKD}$ producing catastrophic results in elderly CKD patients [6]. Previous reported cross sectional cohort studies failed to reveal what factors are more important for progression to ESRD and for occurrence of CVD in these populations [7]. Besides, it still remains uncertain whether the effects of multidisciplinary care are beneficial or not. Two studies correlated multidisciplinary care with modest improvements in survival [8], but another study showed no differences in kidney function or mortality between patients who received multidisciplinary, intensive care and those who did not [6].

Therefore, in the present study, the longitudinal follow-up of elderly patients with stage $4 \mathrm{CKD}$ was carried out in a single center in order to evaluate the characteristics and the prognosis of stage $4 \mathrm{CKD}$ patients $>75$ years in comparison with those between 74 and 65 years and outcome of late nephrology referral in these patients.

\section{Subjects and Methods}

This was a prospective, observational, single-center cohort study. This study was conducted in accordance with the Declaration of Helsinki. Approval for the study was obtained from the Saitama Medical University Ethics Committee, and written informed consent was obtained from each participant.

Patients were recruited from specialist renal clinics at Kidney

*Corresponding author: Hiromichi Suzuki, MD, Ph D, Department of Nephrology, Saitama Medical University, 38 Moroyama-machi, Iruma-gun, Saitama, 350-0495 Japan; Tel: +81-49276-1620; Fax: +81-49295-7338; E-mail: iromichi@saitama-med.ac.jp

Received July 17, 2014; Accepted September 19, 2014; Published September 26, 2014

Citation: Suzuki H, Inoue T, Kikuta T, Hamada Y, Okada H (2014) Entity of Stage 4 CKD over 75 Years Old is Different from that of Less than 75 Years of Age: Characteristics of Elderly Patients with Stage 4 CKD. J Nephrol Ther 4: 183. doi:10.4172/2161-0959.1000183

Copyright: ( 2014 Suzuki H, et al. This is an open-access article distributed under the terms of the Creative Commons Attribution License, which permits unrestricted use, distribution, and reproduction in any medium, provided the original author and source are credited. 
Disease Center, Saitama Medical University from January 2004 to December 2005. All participating patients were followed for 5 years or until death, commencement of renal replacement therapy (RRT), finding of neoplasm, or occurrence of a cardiovascular event (fatal or non-fatal myocardial infarction, cerebrovascular disease, aortic dissection). Accordingly, the main outcomes were death, introduction to RRT, occurrence of neoplasm, and cardiovascular events including fatal and non-fatal disease. Electronic databases were used to obtain information. Inclusion criteria were stage $4 \mathrm{CKD}$ as defined by $\mathrm{K} /$ DOQI [9], not yet on dialysis, followed up for at least 6 months stable renal function within the last 3 months $(<5 \mathrm{~mL} / \mathrm{min} / 1.73 \mathrm{~m} 2$ change in GFR), and no change in medication in the preceding 3 months in our renal specialist clinic. eGFR was calculated using a modified threevariable equation for eGFR in Japanese patients: eGFR=194XAge ${ }^{0.287} \mathrm{XSCr}^{-1.094}(\times 0.739$, if female $)$, where $\mathrm{SCr}=$ serum creatinine $[10]$.

Exclusion criteria included known left ventricular dysfunction (ejection fraction $<50 \%$ ), or signs and symptoms of congestive heart failure, significant valvular or coronary heart disease, and use of sedative or hypnotic drugs or any other drugs potentially affecting blood pressure during ambulatory monitoring, e.g., corticosteroids.

Late referral was defined as the patients who were referred from general physician based clinic during the recruitment of this study.

Diabetes mellitus (DM) was defined as HbAlc $5.8 \%$ or higher or taking anti-diabetics including insulin. Glomerulonephritis (GN) was defined by biopsy or clinical diagnosis such as urine casts including unregulated red cells. The cause of CKD was assessed by reviewing the clinical history and investigations. Patients were classified as "hypertensive/glomerulosclerosis" if the patients had no clear evidence of active renal disease but had a history of hypertensive diseases and positive urinary protein excretion without casts.

Patients were followed every month during the study period. At each clinic visit, serum creatinine, electrolyte concentrations, complete blood count, and other serum chemistries (uric acid, glucose, and liver enzymes) were measured. Chest radiographs and electrocardiography were obtained every year.

During the study, target home blood pressure (BP) was 130/85 mm $\mathrm{Hg}$ or lower and home BP measurements were encouraged [11]. The selection of an antihypertensive agent depended on the physicians' preference. All patients were treated with antihypertensive drugs including renin-angiotensin inhibitors, and blood pressure was wellcontrolled in all patients $[12,13]$.

Subjects were treated with recombinant human erythropoietin (rHuEPO) as necessary and their hemoglobin levels were maintained between 11 to $12 \mathrm{~g} / \mathrm{dL}$ [14]. Subjects were given oral iron supplementation if they were diagnosed with iron deficiency.

Subjects with parathyroid hormone levels greater than $300 \mathrm{pg} / \mathrm{ml}$ were treated with $1,25(\mathrm{OH})_{2} \mathrm{D}_{3}$ and $\mathrm{CaCO}_{3}$ supplements, while patients with levels lower than $70 \mathrm{pg} / \mathrm{ml}$ were treated with $\mathrm{CaCO}_{3}$ to reduce the degree of hyperphosphatemia. Doses were adjusted based on serum levels of calcium and phosphate. Lipid lowering drugs, primarily statin derivatives, were administered if serum cholesterol levels exceeded 240 $\mathrm{mg} / \mathrm{dl}[15]$.

\section{Statistical Analysis}

Statistical analyses were performed using JMP software, version 9 (JMP, A Business Unit of SAS, Cary, NC USA). Values are given as the mean \pm SD. Statistical analysis was performed using Student's "t-test" for comparing means of unpaired variables (or Mann-Whitney tests when applicable). Comparison of more than two sets of unmatched data were performed by one-way of analysis of variance (ANOVA) using the Tukey-test or Kruskal-Wallis tests dependent on the distribution.

Patient event free curves were calculated by the Kaplan-Meier life-table analysis method, and differences between the groups were evaluated by the log-rank test. Subgroups were compared using the $\chi 2$ test for categorical values and continuous variables. $P$ values $<0.05$ were regarded as statistically significant.

\section{Results}

Two hundred and eighty five elderly patients with stage 4 CKD were enrolled in this study. Their baseline characteristics are summarized in the (Table 1). There were no differences in eGFR between the two groups, however, a proportion of the underlying renal diseases were significantly different between the two groups. In the elderly group, the prevalence of diabetic nephropathy was significantly lower $(\mathrm{P}<0.01)$ and that of HT was significantly higher $(\mathrm{P}<0.01)$. In the (Table 2), the hemodynamic variables and laboratory findings are shown. Among these variables, the levels of diastolic blood pressure were significantly lower in patients of the elderly group compared with that of the younger population $(\mathrm{P}<0.05)$. In the (Table 3$)$, there were no significant differences except $\mathrm{HbAlc}$, which was significantly lower in the patients with non-diabetic nephropathy, between the two groups $(\mathrm{P}<0.01)$. In the (Table 4$)$, a comparison of underlying renal disease between the total patients and the patients who were referred during the recruitment period (late referral) is shown. In the late referral patients, the prevalence of diabetic nephropathy were significantly

\begin{tabular}{|c|c|c|}
\hline Variables & Younger Group & Elderly Group \\
\hline Number & 179 & 106 \\
\hline Age (years) & $70.8 \pm 2.0$ & $79.2 \pm 2.6^{*}$ \\
\hline Male (\%) & $106 / 73$ & $58 / 48$ \\
\hline eGFR (ml/min/1.73m²) & $21.6 \pm 3.3$ & $22.1 \pm 4.1$ \\
\hline Underlying disease $($ male/female)(\%) & & \\
\hline DM & $91(60 / 31)(51)$ & $24^{* *}(15 / 7)(23)$ \\
\hline GN & $41(25 / 16)(23)$ & $24(17 / 9)(23)$ \\
\hline HT & $44(18 / 26)(24)$ & $54^{* *}(25 / 29)(51)$ \\
\hline others & $3(3 / 0)(2)$ & $4(1 / 3)(3)$ \\
\hline Late Referral (male/female) $(\%)$ & $44(28 / 16)(25)$ & $23(11 / 12)(22)$ \\
\hline
\end{tabular}

Values are expressed as mean \pm SD. eGFR, estimated glomerular filtration; DM diabetic nephropathy; GN, glomerular nephritis; HT, hypertensive nephrosclerosis. ** indicates $\mathrm{P}<0.01$ compared with younger group.

Table 1: Patients Characteristics.

\begin{tabular}{|c|c|c|}
\hline Variables & Younger group & Elderly group \\
\hline Number & 179 & 106 \\
\hline SBP $(\mathrm{mmHg})$ & $137.5 \pm 13.7$ & $140.9 \pm 18.3$ \\
\hline DBP $(\mathrm{mmHg})$ & $81.9 \pm 6.1$ & $77.9 \pm 6.5^{*}$ \\
\hline HR (beats/min) & $72.3 \pm 8.6$ & $68.3 \pm 5.4$ \\
\hline Serum albumin $(\mathrm{g} / \mathrm{dL})$ & $4.0 \pm 0.3$ & $3.8 \pm 0.6$ \\
\hline Hemoglobin $(\mathrm{g} / \mathrm{dL})$ & $11.6 \pm 0.7$ & $11.2 \pm 0.8$ \\
\hline Total cholesterol $(\mathrm{mg} / \mathrm{dL})$ & $189.2 \pm 42.3$ & $177.2 \pm 46.3$ \\
\hline Phosphate $(\mathrm{mg} / \mathrm{dL})$ & $4.1 \pm 1.7$ & $4.3 \pm 1.5$ \\
\hline Calcium $(\mathrm{mg} / \mathrm{dL})$ & $9.1 \pm 1.1$ & $9.4 \pm 1.0$ \\
\hline Urinary protein excretion $(\mathrm{g} / \mathrm{gCr})$ & $1.34 \pm 0.88$ & $1.23 \pm 0.90$ \\
\hline
\end{tabular}

Values are expressed as mean \pm SD. SBP, systolic blood pressure; DBP, diastolic blood pressure; HR, heart rate.

* indicates $\mathrm{P}<0.05$ compared with younger group.

Table 2: Baseline characteristics of the study population. 


\begin{tabular}{|c|c|c|c|c|}
\hline \multirow[t]{2}{*}{ Variables } & \multicolumn{2}{|c|}{ DM nephropathy } & \multicolumn{2}{|c|}{ Non DM nephropathy } \\
\hline & $75<$ & $75 \geq$ & $75<$ & $75 \geq$ \\
\hline Number & 91 & 24 & 88 & 82 \\
\hline eGFR $(\mathrm{ml} / \mathrm{min} / 1.73 \mathrm{~m}$ & $21.4 \pm 2.3$ & $22.1 \pm 4.0$ & $22.1 \pm 4.1$ & $22.1 \pm 4.5$ \\
\hline $\mathrm{SBP}(\mathrm{mmHg})$ & $121.6 \pm 32.2$ & $133.8 \pm 20.2$ & $136.5 \pm 12.9$ & $135.2 \pm 19.5$ \\
\hline $\mathrm{DBP}(\mathrm{mmHg})$ & $2.4 \pm 7.0$ & $74.4 \pm 16.6^{*}$ & $82.6 \pm 7.2$ & $77.1 \pm 12.5^{*}$ \\
\hline HR (beats/min) & $1.4 \pm 7.9$ & $69.2 \pm 4.5$ & $73.0 \pm 7.6$ & $67.8 \pm 6.3$ \\
\hline Serum albumin $(\mathrm{g} / \mathrm{c}$ & $4.0 \pm 0.3$ & $3 \pm 0.6$ & $4.0 \pm 0.3$ & $8 \pm 0.6$ \\
\hline Hemoglobin ( & $2.4 \pm 1.8$ & $11.2 \pm 0.8$ & $12.8 \pm 0.7$ & $12.6 \pm 0.8$ \\
\hline $\begin{array}{c}\text { Total cholesterol ( } \\
\mathrm{dL} \text { ) }\end{array}$ & +42.3 & 0.3 & 2.7 & $175.1 \pm 38.7$ \\
\hline Phosphate $(\mathrm{mg} / \mathrm{dL})$ & \pm 1.9 & +1.0 & $4.1 \pm<.0$ & $3.5 \pm 0.9$ \\
\hline Calcium (mg/dL) & $2 \pm 0.6$ & $0 \pm 0.5$ & $9.1 \pm 0.7$ & $9.6 \pm 0.4$ \\
\hline $\begin{array}{l}\text { Urinary protein } \\
\text { excretion }(\mathrm{g} / \mathrm{gCr})\end{array}$ & 0.88 & $1.23 \pm 0.90$ & $0.98 \pm 1.22$ & $0.88 \pm 1$ \\
\hline $\mathrm{HbA} 1 \mathrm{C}(\%)$ & $3 \pm 0.4$ & $6.1 \pm 0.6$ & $5.2 \pm 0.3^{\star \star}$ & $5.1 \pm 0.6^{\star \star}$ \\
\hline
\end{tabular}

Values are expressed as mean \pm SD. SBP, systolic blood pressure; DBP, diastolic blood pressure; HR, heart rate.

*indicates $\mathrm{P}<0.05$ compared with the values of each corresponding of younger group and ** indicates $\mathrm{P}<0.01$ compared with the patients with DM nephropathy of each corresponding age.

Table 3: Comparison of variables between DM and non DM nephropathy.

\begin{tabular}{|c|c|c|c|c|}
\hline Variables & \multicolumn{2}{|c|}{ Non late referral } & \multicolumn{2}{c|}{ Late Referral } \\
\hline group & $75<$ & $75 \geq$ & $75<$ & $75 \geq$ \\
\hline Number (\%) & $135(62)$ & $83(38)$ & $44(66)$ & $23(34)$ \\
\hline DM & $56(41)$ & $7(8)$ & $35(80)^{\star *}$ & $17(74)^{\star *}$ \\
\hline GN & $37(32)$ & $21(63)$ & $4(9)^{\star *}$ & $3(12)$ \\
\hline HT & $42(27)$ & $52(27)$ & $2(5)^{\star *}$ & $2(9)^{\star *}$ \\
\hline Others & 0 & $1(2)$ & $3(6)$ & \\
\hline
\end{tabular}

Values are expressed as count (percentage). DM, diabetic nephropathy; GN glomerular nephritis; HT, hypertensive nephrosclerosis. **indicates $\mathrm{P}<0.01$ compared with the values of each corresponding age.

Table 4: Comparison of underlying disease of patients between non late referral population and late referral.

higher and that of GN and HT were significantly lower compared with those of the non-late referral patients $(\mathrm{P}<0.01)$.

The (Table 5) shows a comparison of the hemodynamic variables and laboratory findings between the late nephrology referral patients and those of the non-late referral patients. The levels of serum calcium were significantly lower and those of serum phosphorus were significantly higher $(\mathrm{P}<0.05)$. In the (Table 6$)$, the causes of withdrawal of patients are shown. In both groups, introduction to RRT was the leading cause of withdrawal of the study and in the younger group, the prevalence of introduction to RRT as the cause of withdrawal from the study was significantly higher compared with that in the elderly group $(\mathrm{P}<0.01)$. A Kaplan-Meier curve for event free of the patients is shown in (Figure 1). The incidence-free rates at 1 year and 3 years were $90 \%$ and $81 \%$ in the elderly group and those were $90 \%$ and $57 \%$ in the younger group, respectively. There is a significant difference between the two groups ( $\log$ rank test; $\mathrm{P}=0.008$ ). In the (Table 7 ), comparison of events between the patients with and without diabetic nephropathy is shown. As the total, event free rate were significantly higher in the patients with diabetic nephropathy compared with those without diabetic nephropathy in both younger and elderly groups $(\mathrm{P}<0.05$ and $\mathrm{P}<0.01$, respectively). In addition, in the patients without diabetic nephropathy, introduction to RRT was significantly lower compared with those with diabetic nephropathy in the younger group $(\mathrm{P}<0.05)$. A KaplanMeier curve for event free obtained from the patients with or without diabetic nephropathy is shown in (Figure 2). The incidence-free rates at 1 year and 3 years were $88 \%$ and $80 \%$ in the patients without diabetic nephropathy and those were $87 \%$ and $38 \%$ in the patients with diabetic nephropathy, respectively. There is a significant difference between the two groups (log rank test; $\mathrm{P}=0.003$ ). In the (Table 8 ), a comparison of events between the late referral patients and the non-late referral patients is shown. The rate of introduction to RRT was significantly

\begin{tabular}{|c|c|c|c|c|}
\hline Variables & \multicolumn{2}{c}{ Non late referral } & \multicolumn{2}{c|}{ Late referral } \\
\hline Age (years) & $75<$ & $75 \geq$ & $75<$ & $75 \geq$ \\
\hline Number & 135 & 83 & 44 & 23 \\
\hline Gender (male/female) & $(78 / 57)$ & $(47 / 36)$ & $(28 / 16)$ & $(11 / 12)$ \\
\hline eGFR $\left(\mathrm{mL} / \mathrm{min} / 1.73 \mathrm{~m}^{2}\right)$ & $21.7 \pm 4.3$ & $22.7 \pm 3.9$ & $23.1 \pm 5.1$ & $23.1 \pm 4.9$ \\
\hline SBP $(\mathrm{mmHg})$ & $128.6 \pm 18.2$ & $130.5 \pm 16.3$ & $145.2 \pm 13.1$ & $141.2 \pm 11.9$ \\
\hline DBP $(\mathrm{mmHg})$ & $81.5 \pm 6.7$ & $75.3 \pm 15.9$ & $83.6 \pm 4.9$ & $73.2 \pm 11.4$ \\
\hline HR (beats/min) & $72.3 \pm 6.6$ & $68.1 \pm 4.3$ & $71.6 \pm 7.5$ & $66.6 \pm 7.2$ \\
\hline Serum albumin (g/dL) & $4.1 \pm 0.4$ & $3.9 \pm 0.7$ & $3.6 \pm 0.9$ & $3.8 \pm 1.2$ \\
\hline Hemoglobin $(\mathrm{g} / \mathrm{dL})$ & $12.6 \pm 0.7$ & $12.1 \pm 0.9$ & $11.3 \pm 0.7$ & $11.2 \pm 0.9$ \\
\hline Total cholesterol (mg/dL) & $200.6 \pm 35.3$ & $180.8 \pm 28.7$ & $201.3 \pm 36.7$ & $172.1 \pm 35.3$ \\
\hline Phosphate $(\mathrm{mg} / \mathrm{dL})$ & $3.6 \pm 1.1$ & $3.3 \pm 1.0$ & $5.0 \pm 1.8^{*}$ & $4.8 \pm 0.7^{*}$ \\
\hline Calcium $(\mathrm{mg} / \mathrm{dL})$ & $9.6 \pm 0.5$ & $9.8 \pm 0.3$ & $8.1 \pm 1.1^{*}$ & $8.4 \pm 1.5^{*}$ \\
\hline Urinary protein excretion & $1.12 \pm 0.66$ & $1.00 \pm 0.88$ & $1.42 \pm 1.34$ & $1.31 \pm 1.21$ \\
\hline (g/gCr) & & & & \\
\hline
\end{tabular}

Values are expressed as mean $\pm S D$. * indicates $\mathrm{P}<0.05$ compared with the values of total.

Table 5: Comparison of baseline characteristics between non late referral population and patients with late referral.

\begin{tabular}{|c|c|c|}
\hline Variables & Younger Group & Elderly Group \\
\hline Number & 100 & 35 \\
\hline RRT & $75(75)$ & $15(42)^{\star *}$ \\
\hline Myocardial Infarction & $5(5)$ & $4(12)$ \\
\hline Cerebrovascular Diseases & $5(5)$ & $4(12)$ \\
\hline Heart Failure & $10(10)$ & $6(17)$ \\
\hline Neoplasma & $5(5)$ & $5(14)$ \\
\hline Others & & $1(3)$ \\
\hline
\end{tabular}

Values are expressed as count (percentage). RRT, renal replacement therapy. ${ }^{*}$ indicates $\mathrm{P}<0.01$ compared with younger group.

Table 6: Comparison of events between patients in younger and elderly group.

Kaplan Meier curve of event free for patients in younger and elderly group

Event free

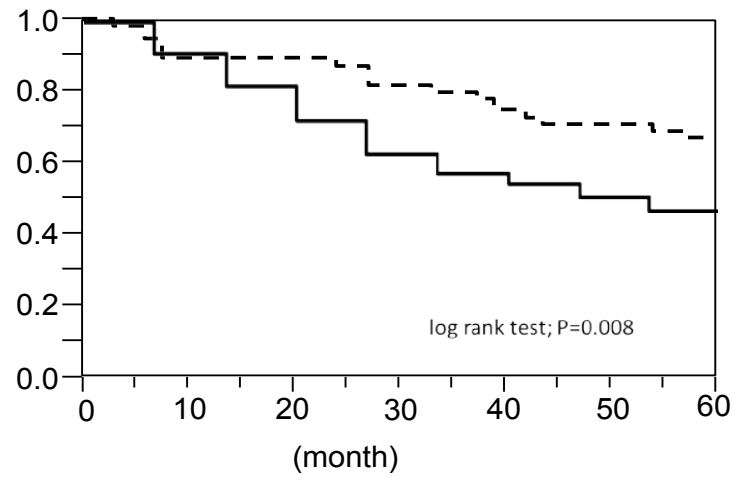

Figure 1: A Kaplan-Meier curve for event free of the patients in the younge and the elderly group. The incidence-free rates at 1 year and 3 years were $90 \%$ and $81 \%$ in the elderly group and those were $90 \%$ and $57 \%$ in the younger group, respectively. There is a significant difference between the two groups (log rank test; $\mathrm{P}=0.008$ ). 
Citation: Suzuki H, Inoue T, Kikuta T, Hamada Y, Okada H (2014) Entity of Stage 4 CKD over 75 Years Old is Different from that of Less than 75 Years of Age: Characteristics of Elderly Patients with Stage 4 CKD. J Nephrol Ther 4: 183. doi:10.4172/2161-0959.1000183

\begin{tabular}{|c|c|c|c|c|}
\hline Variables & DM & & Non DM & \\
\hline Age (years) & $75<$ & $75 \geq$ & $75<$ & $75 \geq$ \\
\hline Number & 91 & 24 & 88 & 82 \\
\hline RRT & $48(53)$ & $10(58)$ & $27^{\star}(64)$ & $5(30)$ \\
\hline Myocardial infarction & $3(5)$ & $2(12)$ & $2(5)$ & $2(12)$ \\
\hline Cerebrovascular disease & $1(2)$ & $2(12)$ & $4(10)$ & $2(12)$ \\
\hline Heart failure & $3(5)$ & $2(12)$ & $7(16)$ & $4(23)$ \\
\hline Neoplamsa & $3(5)$ & $1(6)$ & $2(5)$ & $4(23)$ \\
\hline Total $(\%)$ & $58(64)$ & $17(70)$ & $42^{\star}(48)$ & $17^{\star \star}(21)$ \\
\hline
\end{tabular}

Values are expressed as count (percentage).

DM, diabetic nephrophaty; RRT, renal replacement therapy. * indicates $\mathrm{P}<0.05$ and * *indicates $\mathrm{P}<0.01$ in Comparison with DM group.

Table 7: Comparison of events between patients with diabetic nephropathy and non diabetic nephrophathy.

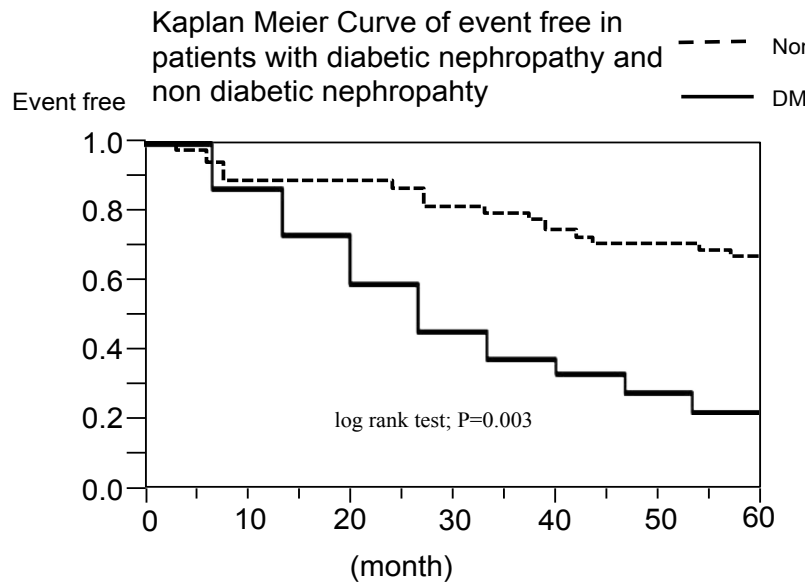

Figure 2: A Kaplan-Meier curve for event free obtained from the patients with or without diabetic nephropathy. The incidence-free rates at 1 year and 3 years were $88 \%$ and $80 \%$ in the patients without diabetic nephropathy and those were $87 \%$ and $38 \%$ in the patients with diabetic nephropathy, respectively. There is a significant difference between the two groups (log rank test; $\mathrm{P}=0.003$ ).

\begin{tabular}{|c|c|c|c|c|}
\hline Variables & \multicolumn{2}{|c|}{ Non late referral } & \multicolumn{2}{c|}{ Late Referral } \\
\hline Age & $75<$ & $75 \geq$ & $75<$ & $75 \geq$ \\
\hline Number (percentage) & $67(70)$ & $28(30)$ & $33(82)$ & $7(18)$ \\
\hline RRT & $45(67)$ & $9(32)$ & $30(91)^{\star *}$ & $6(86)^{\star *}$ \\
\hline Myocardial infaraction & $5(7)$ & $4(14)$ & 0 & 0 \\
\hline Cerebrovascular disease & $3(4)$ & $3(11)$ & $2(6)$ & $1(14)$ \\
\hline Heart failure & $10(15)$ & $6(21)$ & 0 & 0 \\
\hline Neoplasma & $4(7)$ & $5(18)$ & $1(3)$ & 0 \\
\hline Others & 0 & $1(4)$ & 0 & 0 \\
\hline
\end{tabular}

Values are expressed as count (percentage).

RRT, renal replacement therapy. ${ }^{*}$ indicates $\mathrm{P}<0.01$ in comparison with non late referral patients corresponding the same age group.

Table 8: Comparison of events between non late referral population and late referral patients.

higher in the late referral patients compared with those of the non-late referral patients in both the younger and the elderly groups $(\mathrm{P}<0.01)$. A Kaplan-Meier curve for event free obtained from the late referral patients and the non-late referral patients is shown in (Figure 3). The incidence-free rates at 1 year and 3 years were $94 \%$ and $80 \%$ in the total population and those at 1 year and 3 years were $94 \%$ and $64 \%$ in the late nephrology referral patients. There was a significant difference between the two groups $(\mathrm{P}<0.05)$. In the (Table 9$)$, a comparison of causes of introduction to RRT between the patients in the younger and the elderly group is shown. In the patients in the elderly group, the reasons of introduction to RRT due to decreases in eGFR were significantly lower and those in over hydration were significantly higher $(\mathrm{P}<0.01)$ compared with those in the patients in the elderly group.

Selection of modalities for dialysis therapy showed a significant difference between the patients in the younger and the elderly group and between the total population and the late referral patients (Figures 4 and 5). In the patients in the elderly group selected continuous ambulatory peritoneal dialysis (CAPD) therapy more frequently compared with those in the younger group $(\mathrm{P}<0.05)$. However, there were no significant differences in frequency of selection between the late referral patients and the total population.

\section{Discussion}

This long-term prospective observation study provided the following findings. First, event free ratio was significantly higher in the elderly patients compared with the younger patients in stage 4 CKD. Second, diabetic nephropathy had a higher incidence of events compared with the patients without DM nephropathy. Third, late referral produced higher incidence of events compared with the patients regularly cared by nephrologist. Lastly, the elderly patients preferred CAPD as dialysis modality compared with the younger patients.

Age difference in the prognosis observed here probably reflects a variety of different phenomena. The lower incidence of introduction to RRT among the elderly group compared with the younger group

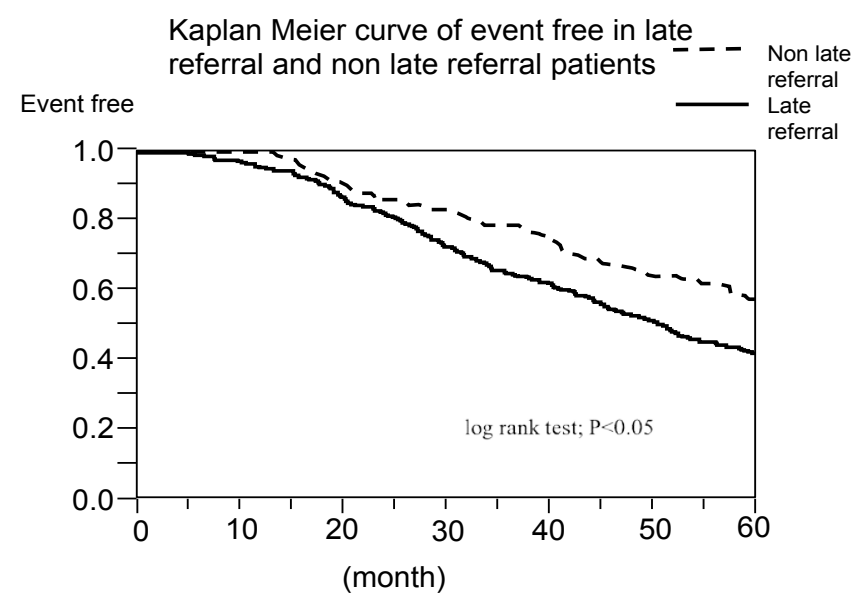

Figure 3: A Kaplan-Meier curve for event free obtained from the late referra patients and the total population. The incidence-free rates at 1 year and 3 years were $94 \%$ and $80 \%$ in the total population and those at 1 year and 3 years were $94 \%$ and $64 \%$ in the late nephrology referral patients. There was a significant difference between the two groups $(P<0.05)$.

\begin{tabular}{|c|c|c|}
\hline Variables & Younger Group & Elderly Group \\
\hline Number & 75 & 15 \\
\hline Decreases in eGFR & $55(73)$ & $4(26)^{\star *}$ \\
\hline Overhydration & $10(13)$ & $6(40)^{\star *}$ \\
\hline Anemia & $1(1)$ & $1(7)$ \\
\hline Increases in Serum Potassium & $1(1)$ & $3(20)$ \\
\hline Anorexia & $8(11)$ & $1(7)$ \\
\hline
\end{tabular}

Values are expressed as count (percentage).

GFR, glomerular filtration rate. Number and $(\%){ }^{* *}$ indicates $\mathrm{P}<0.01$ compared with younger group.

Table 9: Comparison of reasons for introduction of dialysis therapy between younger and elderly patients. 


\section{Selection of Modalities of Dialysis Therapy}

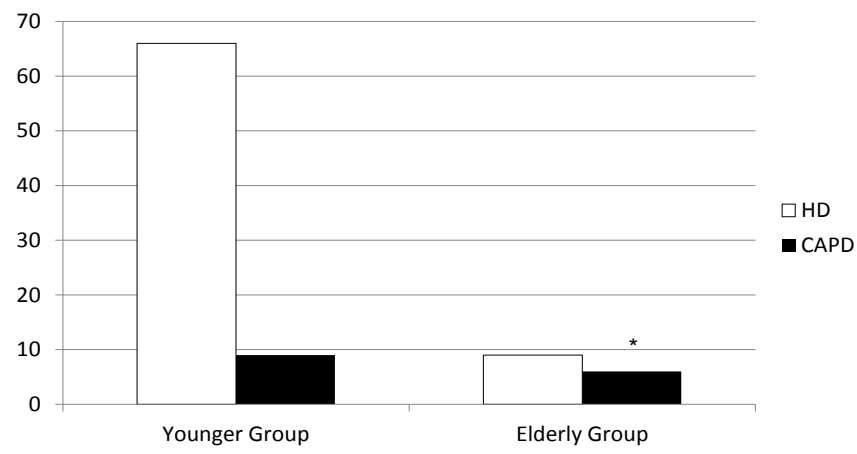

Figure 4: Selection of modalities for dialysis therapy between the patients in the younger and the elderly group. In the patients in the elderly group selected continuous ambulatory peritoneal dialysis (CAPD) therapy more frequently compared with those in the younger group $(\mathrm{P}<0.05)$.

\section{Selection of Modalities}

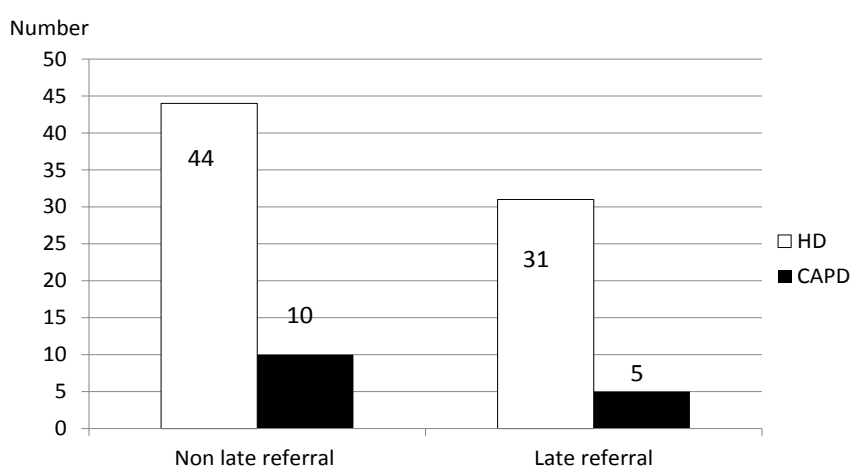

Figure 5: Selection of modalities for dialysis therapy between the total population and the late referral patients. There were no significant differences in frequency of selection between the late referral patients and the non-late referral patients.

with similar levels of eGFR is likely due, at least in part to their greater proportion of diabetic nephropathy as the underlying cause of renal disease. In addition, in the elderly group, the prevalence of HT and GN were significantly greater. These renal diseases are known to be slowly progressive disease $[16,17]$.

Previously, Lindeman [18] reported that the rate of decline of inulin or creatinine clearance increase as patients aged. However, recently O'Hare et al. have demonstrated the contrast findings in that older age was associated with slower decline in eGFR [3]. First, there is 20 years difference between the two studies was carried out. During the two decades, an innovative formula for evaluation of renal function, eGFR, was introduced [19]. This formula is dependent on both age and serum creatinine. If aging produces decreases in muscle mass, eGFR is underestimated. Or conversely, the effects of age on eGFR are overestimated. Combining with these two factors, evaluation of renal function using eGFR formula will need some cautions. Second, several interventions [20] such as antihypertensive agents, especially the renin-angiotensin inhibitors [13], rHuEPO [14], phosphate binders [21] and so on, will decrease morbidity and mortality. These two major factors might relate to slower development to ESRD in the patients in elderly group. Similar findings were previously reported by Eriksen and Ingebretsen [22] demonstrated that $27 \%$ had no decline in eGFR for 10 years in a population of patients average age 75 with a baseline eGFR of $25-55 \mathrm{ml} / \mathrm{min} / 1.73 \mathrm{~m} 2$. In contrast to the reported data, in which the incidence of stage $4 \mathrm{CKD}$ patients were higher than that of development to RRT, the present data showed that CVD was less even in patients with diabetic nephropathy. These data might reflect the incidence of CVD in Japanese general population, in which CVD is less prevalent in proportion of causes of death than that of Western countries $[23,24]$. The proportion of DM was more prevalent in 64-75 patients than patients $>75$. In Japan DM was diagnosed at the age of around 40 years and DM nephropathy is the most prevalent in aged 60 years. Since prognosis of DM nephropathy is expected to be less than 10 years, the patients with DM nephropathy are unable to survive more than 75 years [25]. Besides, in the patients aged $>75$ years, HT is the leading cause of the underlying renal disease in the present study. The cause of nephrosclerosis still remain unclear [26] and some investigators reported that nephrosclerosis is not a product of long term of hypertension [27] and/or atherosclerosis. In elderly CKD 3 to 5 stage patients, there is no decisive renal biopsy proven data for the causes of renal diseases. Generally, the cause of age-related increases in renal fibrosis, which is supposed lead to glomerular sclerosis, interstitial fibrosis, tubular atrophy, vascular sclerosis, and loss of renal function, is poorly understood [3]. Moreover, at the time of when CKD is found in elderly people, the kidneys are already shrunken and nephrogists hesitate to attempt to do renal biopsy.

Recently Smart [28] reported the results of meta-analysis discussing the outcomes of early versus late nephrology referral in CKD patients. In their large meta-analysis, early nephrology referral reduced mortality and hospitalization, better uptake of $\mathrm{PD}$, and earlier placement of arteriovenous fistula for HD in comparison with late nephrology referral. In the present study, a large proportion of the late referral patients were diabetic nephropathy. Moreover, during 5-year observation period, the incidence of introduction to RRT was greater than the total population of the present study. Compared with the other renal specialist clinic, the rate of hospitalization was not counted. However, the rate of selection of dialysis modalities was similar, in which PD was not selected by the late referral patients.

In the present study, we did not count the death rate, because no sudden death was noted and some patients died during hospitalization and some did after introduction to RRT. In these situations, it was very difficult to determine when a patient was dead. With this regard, death rate was not counted.

Several investigators demonstrated that proteinuria was strongly associated with introduction to RRT $[2,29]$, however, our analysis failed to determine a role of proteinuria. This might be due to heterogeneity of underlying renal diseases, because other data were based on population study, in that the prevalence of diabetic nephropathy was less.

\section{Study Limitations}

First, this study was conducted in a single center and the results may be less generalizable to other settings. However, the early nephrology referral would be recommended and moderately advanced CKD elderly patients, evaluation of eGFR must be cautious. Second, the number of participated patients was small and more number of these patients is absolutely needed to finalize our current findings. Third, in the present study, patients with hypertension and renal failure without evidence of primary renal disease or DM nephropathy are intentionally diagnosed as having hypertensive renal disease. 
Citation: Suzuki H, Inoue T, Kikuta T, Hamada Y, Okada H (2014) Entity of Stage 4 CKD over 75 Years Old is Different from that of Less than 75 Years of Age: Characteristics of Elderly Patients with Stage 4 CKD. J Nephrol Ther 4: 183. doi:10.4172/2161-0959.1000183

Recently since incidence of ischemic nephropathy is suggested to be higher than previously reported [30,31], possibilities of renovascular disease due to atherosclerosis should be considered. In the present study, this diagnosis was not applicable to the participated patients. In spite of these several limitations, this study was carried out in a single center cancelling out the heterogeneity produced by center-to-center interactions.

In summary, this 5-year prospective observation study demonstrated that, if elderly people $>65$ years is divided into $2 ; 65-74$ and $>75$ years in CKD patients, a proportion of the underlying renal disease was completely different. In the elderly patients $>75$ years, HT nephrosclerosis and GN were predominant. In contrast, in the patients 65 to 74 years, diabetic nephropathy was predominant. In addition, the ratio of development to RRT was higher in the patients 65 to 74 years, probably being due to a large proportion of diabetic nephropathy as the leading cause of renal disease. Second, a prognosis of diabetic nephropathy, regardless of age groups, was poor. Third, a prognosis of the late referral patients was also not well.

From these findings, it is concluded that in the patients $>75$ years, the values of eGFR may be overestimated and a prognosis of diabetic nephropathy was poor. And lastly, the early nephrology referral is encouraged.

\section{Conflict of Interest} study.

The authors declare that they have no conflicts of interest in this

\section{Acknowledgement}

Mrs Sachiko Nakazato, a secretary, calculated the data and typed the manuscript.

\section{References}

1. Imai E, Matsuo S, Makino H, Watanabe T, Akizawa T, et al. (2010) Chronic Kidney Disease Japan Cohort study: baseline characteristics and factors associated with causative diseases and renal function. Clin Exp Nephrol 14: $558-570$.

2. Obi Y, Kimura T, Nagasawa Y, Yamamoto R, Yasuda K, et al. (2010) Impact of age and overt proteinuria on outcomes of stage 3 to 5 chronic kidney disease in a referred cohort. Clin J Am Soc Nephrol 5: 1558-1565.

3. O'Hare AM, Choi Al, Bertenthal D, Bacchetti P, Garg AX, et al. (2007) Age affects outcomes in chronic kidney disease. J Am Soc Nephrol 18: 2758-2765.

4. Iseki K (2010) Renal outcomes in chronic kidney disease. Nephrology (Carlton) 15 Suppl 2: 27-30.

5. Maccariello E, Rocha E, Valente C, Nogueira L, Rocha PT, et al. (2008) Effects of early changes in organ dysfunctions on the outcomes of critically ill patients in need of renal replacement therapy. Clinics (Sao Paulo) 63: 343-350.

6. Harris LE, Luft FC, Rudy DW, Kesterson JG, Tierney WM (1998) Effects of multidisciplinary case management in patients with chronic renal insufficiency. Am J Med 105: 464-471.

7. Herzog CA, Asinger RW, Berger AK, Charytan DM, Díez J, et al. (2011) Cardiovascular disease in chronic kidney disease. A clinical update from Kidney Disease: Improving Global Outcomes (KDIGO). Kidney Int 80: 572-586.

8. Hemmelgarn BR, Manns BJ, Zhang J, Tonelli M, Klarenbach S, et al. (2007) Association between multidisciplinary care and survival for elderly patients with chronic kidney disease. J Am Soc Nephrol 18: 993-999.

9. National Kidney Foundation (2002) K/DOQI clinical practice guidelines for chronic kidney disease: evaluation, classification, and stratification. Am J Kidney Dis 39: S1-266.

10. Matsuo S, Imai E, Horio M, Yasuda Y, Tomita K, et al. (2009) Revised equations for estimated GFR from serum creatinine in Japan. Am J Kidney Dis 53: 982

11. Suzuki H, Moriwaki K, Nakamoto H, Sugahara S, Kanno Y,et al.(2001) Blood pressure reduction in the morning yields beneficial effects on progression of chronic renal insufficiency with regression of left venricular hypertrophy. Clinical and Experimental Hypertension.

12. Suzuki H, Saruta T (2004) An overview of blood pressure regulation associated with the kidney. Contrib Nephrol 143: 1-15.

13. Suzuki H (2004) Angiotensin type 1 receptor blockers in chronic kidney disease Contrib Nephrol 143: 159-166.

14. Matsuda A, Kitaoka T, Suzuki H (2014) Effect of hemoglobin targe on progression of chronic kidney disease. Journal of Nephrology \& Therapeutic S1:006

15. Suzuki $H$, Watanabe $Y$, Kumagai $H$, Shuto $H(2013)$ Comparative efficacy and adverse effects of the addition of ezetimibe to statin versus statin titration in chronic kidney disease patients. Ther Adv Cardiovasc Dis 7: 306-315.

16. Evans M, Fryzek JP, Elinder CG, Cohen SS, McLaughlin JK, et al. (2005) The natural history of chronic renal failure: results from an unselected, populationbased, inception cohort in Sweden. Am J Kidney Dis 46: 863-870.

17. Hemmelgarn BR, Zhang J, Manns BJ, Tonelli M, Larsen E, et al. (2006) Progression of kidney dysfunction in the community-dwelling elderly. Kidney Int 69: 2155-2161.

18. Lindeman RD, Tobin J, Shock NW (1985) Longitudinal studies on the rate of decline in renal function with age. J Am Geriatr Soc 33: 278-285.

19. Lamb EJ, Webb MC, O'Riordan SE (2007) Using the modification of diet in renal disease (MDRD) and Cockcroft and Gault equations to estimate glomerular filtration rate (GFR) in older people. Age Ageing 36: 689-692.

20. Thomsen S (2013) Delayed progression to dialysis with early and intensive management of predialysis chronic kidney disease: a case-based approach. Case Rep Nephrol Urol 3: 74-86.

21. Ketteler M, Biggar PH (2013) Use of phosphate binders in chronic kidney disease. Curr Opin Nephrol Hypertens 22: 413-420.

22. Eriksen BO, Ingebretsen OC (2006) The progression of chronic kidney disease a 10 -year population-based study of the effects of gender and age. Kidney Int 69: $375-382$

23. Kubo M, Kiyohara Y, Kato I, Tanizaki Y, Arima H, et al. (2003) Trends in the incidence, mortality, and survival rate of cardiovascular disease in a Japanese community: the Hisayama study. Stroke 34: 2349-2354.

24. Ueshima H (2007) Explanation for the Japanese paradox: prevention of increase in coronary heart disease and reduction in stroke. $J$ Atheroscler Thromb 14: 278-286.

25. Takane H, Kanno Y, Ohno Y, Sugahara S, Suzuki H (2007) Salt and excess food intake produced diabetic nephropathy in Japan. Contrib Nephrol 155 125-135.

26. Luft FC (2004) Hypertensive nephrosclerosis: update. Curr Opin Nephrol Hypertens 13: 147-154.

27. Rosario RF1, Wesson DE (2006) Primary hypertension and nephropathy. Curr Opin Nephrol Hypertens 15: 130-134.

28. Smart NA, Dieberg G, Ladhani M, Titus T (2014) Early referral to specialist nephrology services for preventing the progression to end-stage kidney disease. Cochrane Database Syst Rev 6: CD007333.

29. Nerpin E, Ingelsson E, Risérus U, Sundström J, Larsson A, et al. (2011) The combined contribution of albuminuria and glomerular filtration rate to the prediction of cardiovascular mortality in elderly men. Nephrol Dial Transplant 26: $2820-2827$

30. Park S, Jung JH, Seo HS, Ko YG, Choi D, et al. (2004) The prevalence and clinical predictors of atherosclerotic renal artery stenosis in patients undergoing coronary angiography. Heart Vessels 19: 275-279.

31. Textor SC, Misra S, Oderich GS (2013) Percutaneous revascularization for ischemic nephropathy: the past, present, and future. Kidney Int 83: 28-40. 